\section{Pimecrolimus Plus Localized Narrow Band 311nm UVB (Microphototherapy) as a Treatment of Localized Vitiligo}

\author{
Mohamed Amer ${ }^{1,2}$ and Amin Amer ${ }^{1 *}$ \\ ${ }^{1}$ Amer skin care centers, Cairo, Egypt \\ ${ }^{2}$ Dermatology and Venereology, Medical School, Zagazig University, Cairo, \\ Egypt
}

\begin{abstract}
Background: Vitiligo is an autoimmune common disease that produces disfiguring white patches of depigmentation (milk color). Many therapies are often not very effective and needs a long period of time for acceptable results.

Objective: We sought of trying different methods of treatment and evaluate the best of them for Vitiligo patients.

Methods: Ninety patients were collected and divided into three groups and three different methods of treatment were given to each individual. Topical Pimecrolimus was given to group $\mathrm{C}$, localized Narrow band $311 \mathrm{~nm}$ UVB (Microphototherapy) was given alone to group $A$ while a mix of them were given to group $B$.

Results: The best results were seen in group $B$ which was given Microphototherapy plus Pimecrolimus and after 6 months 10 patients showed full repigmentation and 8 patients showed more than $75 \%$ improvement, 10 showed $50 \%$ improvement two patients showed no change at all while 5 patients showed recurrence after improvement. No pain was reported by patients while itching and burning sensation was reported by 17 patients out of 30 in group $B$. The average cumulative UV-B dose within the treatment was 6.025 $\mathrm{J} / \mathrm{cm}^{2}$ (range of $3.4-7 \mathrm{~J} / \mathrm{cm}^{2}$ ) per subject.

Conclusion: Topical Pimecrolimus plus Microphototherapy is an effective therapy in localized vitiligo.
\end{abstract}

\section{Introduction}

Vitiligo is a common acquired, idiopathic skin disorder characterized by one or more patches of skin depigmentation caused by

\footnotetext{
*Corresponding author: Amin Amer, Amer skin care centers, Cairo, 86 Ahmed El Zayyat Street, Dokki, Cairo, Egypt, Tel: +20 122223133; E-mail: aminamer74@gmail.com

Citation: Amer M, Amer A (2016) Pimecrolimus Plus Localized Narrow Band $311 \mathrm{~nm}$ UVB (Microphototherapy) as a Treatment of Localized Vitiligo. J Clin Dermatol Ther 3: 019.

Received: November 03, 2015; Accepted: May 12, 2016; Published: June 04, 2016
}

destruction of cutaneous melanocytes. The precise cause of melanocytes destruction is unknown, potential pathogenic mechanisms include genetic, neural, biochemical, self destructive, viral, and autoimmune mechanisms [1-6].

Vitiligo is characterized clinically by the development of depigmented macules and patches that correspond histologically to decrease or absent cutaneous melanocytes. The most accepted mechanism of melanocytes destruction in vitiligo is theorized to be an autoimmune, lymphocytic attack on melanocytes [7].

Vitiligo affects the skin with many negative impacts on the quality of life of affected individuals and between 1-2 \% of the world population without any racial, geographic or sex differences are affected [8].

In this present study we are trying to figure out or to find the best way which can give the best outcome for treatment of such physiological disease.

Although UV rays are beneficial in vitiligo treatment yet it is not without hazards [9]. The advantage of using Microphototherapy on regular UVB machines is to deliver the UVB Rays just to the target area.

Our study is concerned with evaluation of its efficacy in treating vitiligo with or without using another immunomodulater.

\section{Patients and Methods}

This work was done in Amer skin care centers (Zagazig and Cairo) from May 2008 to March 2014 and patients were selected on the following criteria:

- Age ranges between 15-65 years either female or male.

- No given treatment for at least 2 months before their participation in the study.

- Stable vitiligo for at least the past 3 months before the study.

Ninety patients were selected and divided randomly into three groups (group A, B, C).

Group A were treated with Microphototherapy (localized UVB narrowband $311 \mathrm{~nm}$ ) three times weekly for 16 weeks.

Group B were treated with Microphototherapy (localized UVB narrowband $311 \mathrm{~nm}$ ) three times weekly with applying topical Pimecrolimus every day except on the session day for 16 weeks.

Group C were treated by applying Pimecrolimus topically treatment twice daily only for 16 weeks,

Patients of all groups were evaluated monthly for 4 months, so patients were evaluated on week 4, 8, 12 and 16 and after 6 months. 
Citation: Amer M, Amer A (2016) Pimecrolimus Plus Localized Narrow Band 311nm UVB (Microphototherapy) as a Treatment of Localized Vitiligo. J Clin Dermatol Ther 3: 019.

- Page 2 of $4 \cdot$

Evaluation of response to treatment was evaluated according to the following guide:

- Excellent

- Very good

- Good

- Fair

- No Response

All patients were photographed before, during, and after treatment (Figures 1-4).

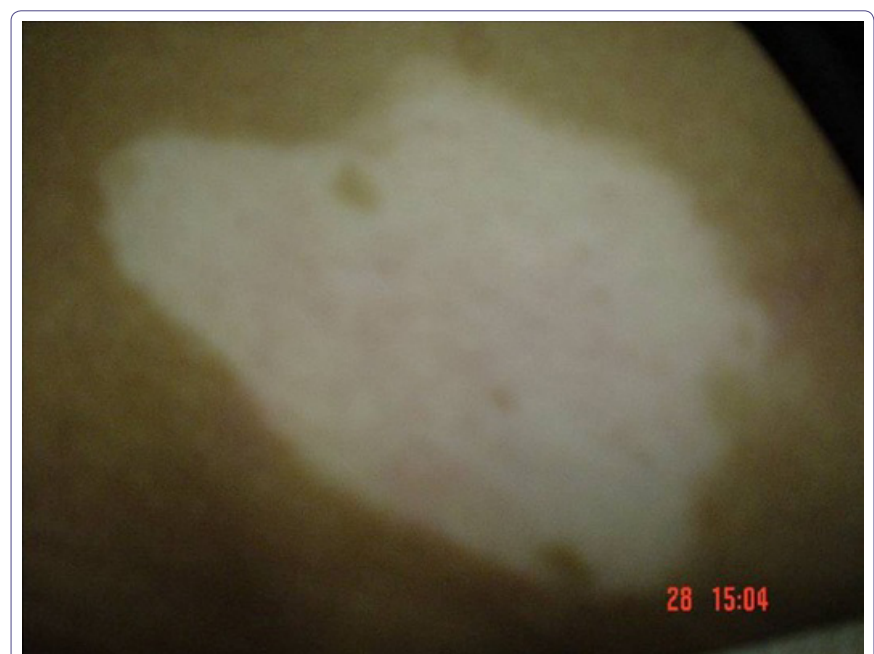

Figure1: Localized vitiligo in the back before treatment.

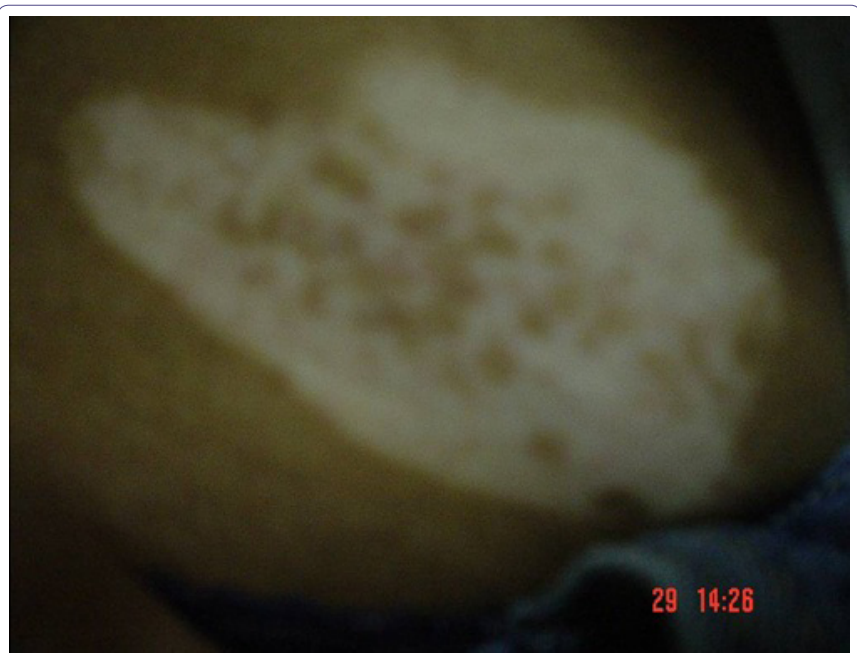

Figure 2: Back after 2 months of treatment (Microtehrapy and Pimecrolimus).

\section{Methods}

\section{Ratok ${ }^{\circledR}$ therapy device}

Ratok $^{\star}$ therapy employs an exclusive device (covered by international patent and currently produced by Quanta System Spa) that supplies UVB rays with emission peak of $311 \mathrm{~nm}$. It can vary the "light power" and the ray dimensions to consequently hit depigmented epidermic patches of specific shape and size, without interfering with the normally-pigmented skin. The spot duration varies from 1 to

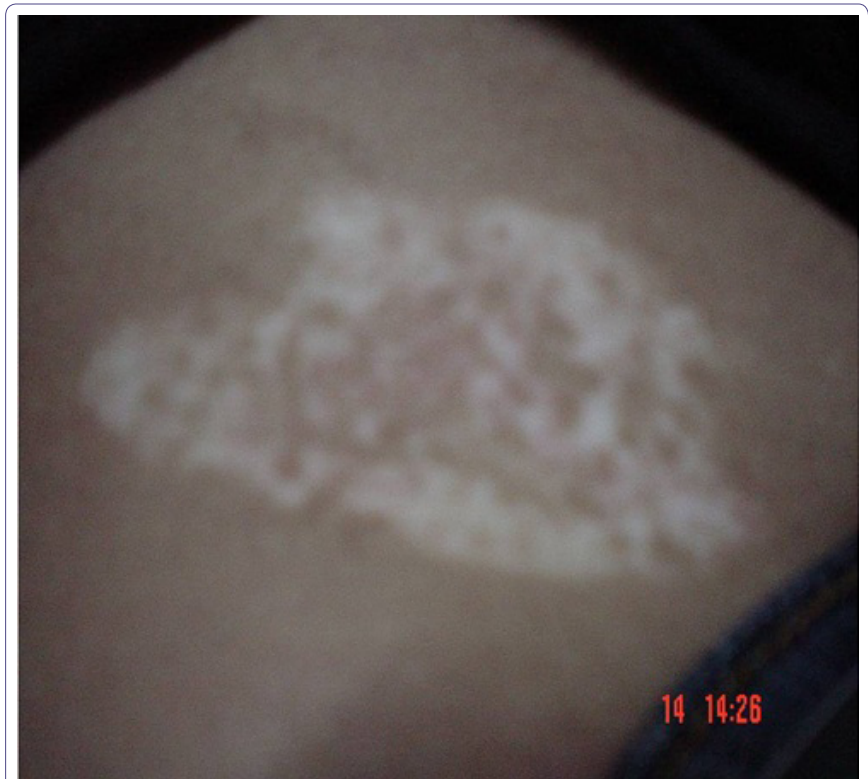

Figure 3: Back after 3 months of treatment.

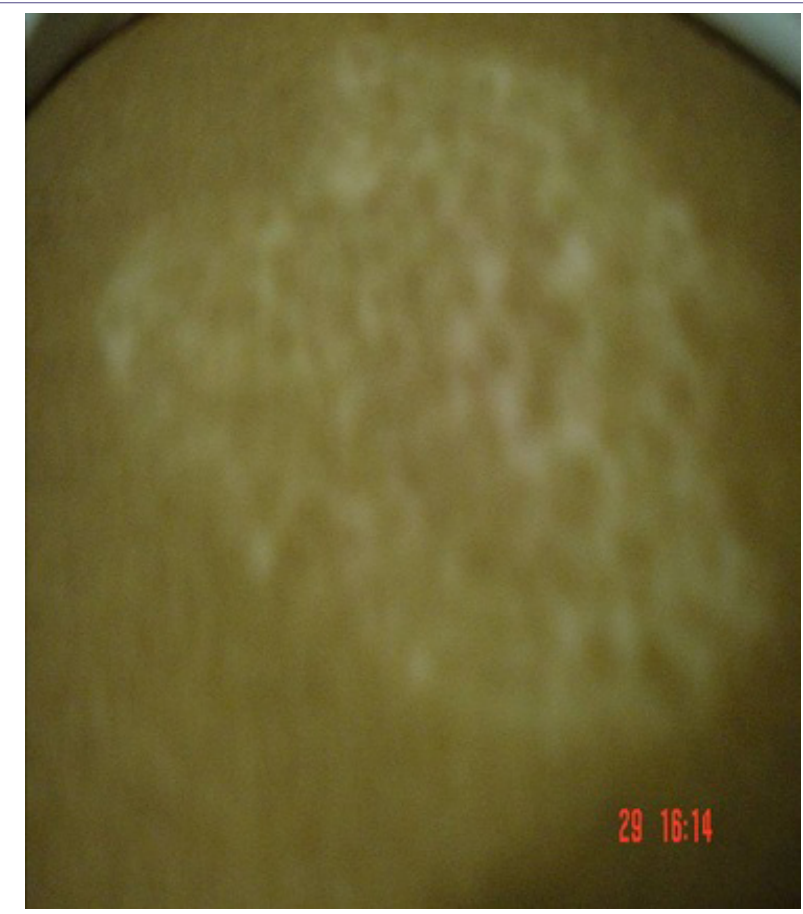

Figure 4: Back after 5 months.

3 seconds and, as a consequence of the stimulation, 12/24 hours later a uniform and/or multiform erythematous reaction appears.

Effect obtained using specific micro-perforated molds (masks) of few millimetres diameter, applied directly on the skin to be treated. The chromatic reconstruction of vitiligo patches does not happen by peripheral narrowing, but by progressive melanic filling of the entire skin surface affected by achromia. For better results a skin moisturizer was applied before directing the localized UVB to the skin.

Pimecrolimus which is a calcineurin inhibitor was applied to 30 patients combined with localized Microphototherapy (Group B) and to other 30 patients alone (Group C). 


\section{Results}

All groups showed improvement with different percentage after treatment and group B showed the best results while groups A\&C were moderately improved.

Group B showed very good to excellent improvement of patients with a percentage of $75 \%$ while group A showed good to very good improvement and finally group C showed fair to good improvement.

The main concern of patients was the longtime of treatment, the high cost of sessions and topical treatment. Slow response of treatment was the other concern of patients which made them suffer more psychologically and even thought of discontinuing sessions. The percentage of improvement of group B which was a mix of (Microphototherapy and Pimecrolimus) exceeded 75\%.

In group B after 6 months 10 patients showed full repigmentation and 8 patients showed more than $75 \%$ improvement while 10 patients showed 50\% improvement and two patients showed no change at all while 5 patients showed recurrence after improvement. No pain was reported by patients while itching and burning sensation was reported by 17 patients out of 30 in group B. The average cumulative UV-B dose within the treatment was $6.025 \mathrm{~J} / \mathrm{cm}^{2}$ (range of $3.4-7 \mathrm{~J} / \mathrm{cm}^{2}$ ) per subject.

Group A showed good to very good improvement with repigmentation up to $50 \%$ improvement. Twelve patients showed up to $70 \%$ improvement while 14 patients showed good improvement and 4 patients showed no improvement and 7 patients after improvement recurrence occurred after 7 months of treatment.

Group C which was treated with calcineurin inhibitor alone showed a variation of results and 8 patients showed very good improvement, 7 patients showed good improvement, 10 patients showed fair improvement and 5 with no changes at all. 8 patients showed recurrence after few months of treatment.

\section{Discussion}

Evidence based medicine metanalysis of all studies recently showed that the highest mean success rates in repigmentation where achieved with narrow band UVB. No side effects were reported with UVB therapy [10] For patients with localized vitiligo (less than $20 \%$ of total body surface affected), UVB can be delivered directly to the lesions by what is known as microphototherapy even though a class III corticosteroid is advised as first choice therapy [10].

In group A 18 out of 30 patients showed very good improvement on localized UVB treatment which led us to suggest that localized UVB therapy may be considered the first choice therapy for localized vitiligo. This may reduce the psychological impact on vitiligo patients especially those who suffers from their face affection of the disease which is a finding that deserves further studies.

Many studies showed the efficacy of calcineurin inhibitors (Pimecrolimus and Tacrolimus) [11-17].

In one study sixteen patients with vitiligo, who attend the Baskent University Dermatology Department, Ankara, Turkey. In each patient, two lesions similar to each other in size and time of evaluation were selected to apply either $1 \%$ Pimecrolimus or $0.05 \%$ clobestasol propionate twice a day for 8 weeks in a double -bind randomized way. Patients were instructed to apply sun screens.

Patients were evaluated by a blind investigator every 2 weeks for repigmentation and adverse effects. Percentage of repigmentation was analyzed by calculating the area of pigmentation at the end of the study. The lesions were drawn on the transparent sheet before and after treatment. The areas of pigmentation were measured by using the $1-\mathrm{mm}^{2}$ graded paper. The mean percentage of repigmentation was $57.7 \%$ for clobestasol and $32.1 \%$ for Pimecrolimus. This difference was statistically significant [11-17].

In current study the patients in Group C (30 patients) 7 showed a good response patients and 10 with fair response. This results showed less efficacy compared to UVB on the other hand the combined therapy including localized UVB treatment and calcineurin inhibitor (Pimecrolimus) in Group B showed the best results obtained in the three groups of patients treated in current study.

In a study using microphototherapy alone for 730 patients with vitiligo (segmental and non segmental) were treated for 12 months achieved normal repigmentation for more than $75 \%$ in the treated area in $69.48 \%$ of the patients, In particular 112 were totally repigmented . One hundred and fifty five $(21.12 \%)$ individuals achieved 50-75\% pigmentation of the treated areas, and 69 (9.40\%) showed less than $50 \%$ repigmentation. No patients showed acute or chronic relevant adverse effects.

In conclusion combined treatment with both targeted UVB Microphototherapy and calcineurin inhibitors (Pimecrolimus) may be considered as first line treatment in vitiligo that affects less than $10 \%$ of skin surface.

In our opinion the available data in this work should encourage for further studies using this combined treatment for localized vitiligo.

Also targeted UVB therapy can be tried alone in some selected cases of less than $10 \%$ skin affection.

\section{References}

1. Grimes PE (2003) XV Disorders of pigmentation. In: Dale DC, Federman DD (eds.). Dermatology. Scientific American(r) Medicine, WebMD Inc., New York City, USA Pg no: 526-534.

2. Bystryn JC (1997) Immune mechanisms in vitiligo. Clin Dermatol 15: 853-861.

3. Nordlund JJ, Majumder PP (1997) Recent investigations on vitiligo vulgaris. Dermatol Clin 15: 69-78.

4. Lerner $A B(1971)$ On the etiology of vitiligo and gray hair. Am J Med 51: $141-147$.

5. Al'Abadie MS, Senior HJ, Bleehen SS, Gawkrodger DJ (1994) Neuropeptide and neuronal marker studies in vitiligo. Br J Dermatol 131: 160-165.

6. Bystryn JC (1989) Serum antibodies in vitiligo patients. Clin Dermatol 7: 136145.

7. Silverberg NB, Lin P, Travis L, Farley-Li J, Mancini AJ, et al. (2004) Tacrolimus ointment promotes repigmentation of vitiligo in children: a review of 57 cases. J Am Acad Dermatol 51: 760-766.

8. Lotti TM, Menchini G, Andreassi L (1999) UV-B radiation microphototherapy. An elective treatment for segmental vitiligo. J Eur Acad Dermatol Venereol 13: $102-108$.

9. Kovacs SO (1998) Vitiligo. J Am Acad Dermatol 38: 647-666.

10. Njoo MD, Spuls PI, Bos JD, Westerhof W, Bossuyt PM (1998) Nonsurgical repigmentation therapies in vitiligo. Meta-analysis of the literature. Arch Dermatol 134: 1532-1540.

11. Lepe V, Moncada B, Castanedo-Cazares JP, Torres-Alvarez MB, Ortiz CA, et al. (2003) A double-blind randomized trial of $0.1 \%$ tacrolimus vs $0.05 \%$ clobetasol for the treatment of childhood vitiligo. Arch Dermatol 139: 581-585.

12. Coskun B, Saral Y, Turgut D (2005) Topical $0.05 \%$ clobetasol propionate versus $1 \%$ pimecrolimus ointment in vitiligo. Eur $\mathrm{J}$ Dermatol 15: 88-91. 
Citation: Amer M, Amer A (2016) Pimecrolimus Plus Localized Narrow Band 311nm UVB (Microphototherapy) as a Treatment of Localized Vitiligo. J Clin Dermatol Ther 3: 019.

- Page 4 of $4 \cdot$

13. Kanwar AJ, Dogra S, Parsad D (2004) Topical tacrolimus for treatment of childhood vitiligo in Asians. Clin Exp Dermatol 29: 589-592.

14. Travis LB, Weinberg JM, Silverberg NB (2003) Successful treatment of vitiligo with $0.1 \%$ tacrolimus ointment. Arch Dermatol 139: 571-574.

15. Grimes PE, Morris R, Avaniss-Aghajani E, Soriano T, Meraz M, Metzger A et al. (2004) Topical Tacrolimus therapy for vitiligo: therapeutic responses and skin messenger RNA expression of proinflammatory cytokines. J Am Acad Dermatol 51: 52-61.
16. Grimes PE, Soriano T, Dytoc MT (2002) Topical tacrolimus for repigmentation of vitiligo. J Am Acad Dermatol 47: 789-791.

17. Tanghetti EA (2003) Tacrolimus ointment $0.1 \%$ produces repigmentation in patients with vitiligo: results of a prospective patient series. Cutis 71: 158-162. 